\title{
La práctica pedagógica desde las situaciones a-didácticas en matemáticas
}

\section{The pedagogical practice since adidactic situations in mathematics}

\author{
Alfonso Jiménez-Espinosa' \\ Daysy Maite Sánchez-Bareño ${ }^{2}$
}

Recibido: octubre 18 de 2018

Aceptado: diciembre 09 de 2018

\begin{abstract}
Resumen
El artículo tiene como objetivo caracterizar la práctica pedagógica a partir del uso de situaciones a-didácticas en clases de matemáticas. La investigación tuvo un enfoque cualitativo, bajo la técnica de investigación acción, con el uso de instrumentos como: observación no participante, cuestionario de pregunta abierta, diario de campo del investigador, grupos focales y diseño y aplicación de situaciones a-didácticas específicas. La población objeto de estudio fue de 4 profesores y sus estudiantes, pertenecientes al nivel de educación básica. Los resultados indican que cuando el profesor confronta la visión de su clase con lo observado por otros, y mediado por la reflexión colectiva sobre su práctica, comienza a tener otra actitud y otra forma de ver la dinámica de una clase. Igualmente, la implementación de las situaciones a-didácticas mejora la dinámica de la clase de matemáticas; la motivación y el entusiasmo, son algunas de las emociones expresadas por los estudiantes hacia el aprendizaje, dejando en evidencia la importancia de innovar, de generar desafíos y desequilibrios, para que la enseñanza de las matemáticas sea más que solo repetir los contenidos y mecanizarlos.
\end{abstract}

Palabras clave: práctica pedagógica, matemáticas, aprendizaje, situaciones a-didácticas.

\begin{abstract}
The aim of this article is to characterize the pedagogical practice from the use of non-didactic situations in Mathematics classes. This research uses a qualitative approach, under the technique of action research, with the use of instruments such as: non-participant observation, open-ended questionnaire, researcher's field diary, focus groups and design and application of specific non didactic situations. The population studied was made up of 4 teachers and their students who belong to basic education. When the teacher faces the vision of his class and the same class being observed by others, mediated by the collective reflection on his practice; he begins to have another attitude and another way of seeing the dynamics of a class. Likewise, the implementation of non-didactic situations improves the dynamics of the math class. Motivation and enthusiasm are some of the emotions expressed by students towards learning, revealing the importance of innovating, generating challenges and imbalances, so that the teaching of Mathematics is more than just repeating the contents and mechanizing them.
\end{abstract}

Keywords: pedagogical practice, mathematics, learning, non-didactic.

1 Licenciado en Matemáticas y Física, Doctor en Educación Matemática, Universidad Pedagógica y Tecnológica de Colombia, Tunja, Colombia.E-mail: alfonso.jimenez@uptc.edu.co

2 Licenciada en Matemática, Estudiante de Maestría en Educación Matemática, Institución Educativa San Pedro Claver, Chitaraque, Colombia. E-mail: mayday301@hotmail.com 


\section{Introducción}

En Colombia desde finales de la década de los ochenta, el Estado puso entre sus prioridades el mejoramiento de la calidad de la educación, generando políticas encaminadas a buscar una educación de alta calidad, pidiendo a los profesores replantear aspectos que afectan directamente al contexto educativo. Esto se evidenció en el gobierno de Juan Manuel Santos Calderón (20142018), que colocó como slogan el “llegar a ser uno de los países más educados", al año 2025. Sin embargo, estas políticas no siempre son tan eficientes, o simplemente se quedan en el papel, pues no se dan las condiciones plenas a los profesores y a las instituciones para alcanzar esa pretendida alta calidad (Riscanevo-Espitia, 2016).

La práctica pedagógica de los docentes está inmersa en un sistema educativo que también ha sido objeto de muchas críticas, revisiones y reestructuraciones. Al respecto, Báez, Cantú y Gómez (2007), expresan: “la práctica de enseñanza (...), se ha visto fuertemente cuestionada por investigaciones y la sociedad en general, en función de los resultados de los procesos educativos. Se discute sobre la calidad de la práctica docente y de la educación...."

Los resultados de pruebas censales, como Saber, que el Estado aplica a los estudiantes en los niveles 3, 5, 9 y 11, para examinar la calidad de la educación, parecen indicar que la escuela poco favorece el desarrollo del pensamiento matemático (MEN, 2017). En contraste, se favorecen aprendizajes algo mecánicos y algorítmicos, ya que frente a estos últimos los estudiantes tienen un buen nivel de respuesta; no así, cuando se trata de resolver problemas, y aún menos, si son problemas sobre situaciones en contexto (Vesga-Bravo \& Escobar-Sánchez, 2018).

Según Corts y De la Vega (2004), algunos hechos muestran que la capacidad de los alumnos de dar respuestas inteligentes se trunca debido a la manera como interviene el docente habitualmente, cuando los alumnos no contestan la respuesta esperada. Igualmente, Cantoral y Farfán (2003), indican que es necesario atender aspectos que los profesores de matemáticas poco contemplan, como la influencia de las propias acciones del profesor en los actos de aprendizaje de sus alumnos, o la forma en que el favorecimiento del diálogo interviene en el desarrollo del pensamiento matemático.

La investigación aquí reportada, se preguntó por las características de la práctica pedagógica a partir del uso de situaciones a-didácticas para el aprendizaje de temas específicos de matemáticas, en educación básica. En este sentido, se toman como referentes la práctica pedagógica y la Teoría de las Situaciones Didácticas, ya que entendemos que la reflexión sobre la práctica que lleve a la (re) significación (Jiménez-Espinosa, 2002), y la elección de buenas situaciones de aprendizaje, son la clave para generar los aprendizajes matemáticos de los estudiantes, pretendidos por el profesor (Godino, 2003).

La investigación tuvo un enfoque cualitativo, bajo la técnica de investigación acción, con el uso de instrumentos como observación no participante, cuestionario de pregunta abierta, diario de campo del investigador, grupos focales y diseño y aplicación de situaciones a-didácticas específicas, referidas a la temática seleccionada. A continuación se presenta el fundamento teórico de la práctica pedagógica y las situaciones didácticas, junto con la metodología seguida en el trabajo. Luego se presentan los resultados y se brindan las conclusiones del estudio.

\section{Marco teórico y metodología}

\subsection{La práctica Pedagógica}

Serres (2007), define la práctica pedagógica como las "acciones intencionadas que realiza el profesor con base en sus conocimientos, experiencias y formación académica, referidas antes, durante y después de la clase, y se consideran un trabajo cí- 
clico, pues incluyen la planificación, la ejecución y la evaluación del mismo".

Para Díaz (2006), la práctica pedagógica es la actividad diaria que desarrollan los profesores en sus aulas y fuera de estas, que son orientadas por el currículo, y con el propósito de la formación de sus alumnos. Estas actividades están permeadas por componentes didáctico-pedagógicos, de alguna forma orientados por los fines y principios de formación integral que establece el currículo, pero también por sus creencias y concepciones. En cuanto a la importancia de investigar la práctica pedagógica, autores como Sola (2004) destaca que la práctica es tanto el motor como la condición fundamental para repensar la formación docente, pues es la única posibilidad de crear conocimiento profesional sobre la práctica y se debe hacer en torno a la práctica concreta de aula.

Otros autores, como Escobar (2007), no habla de práctica pedagógica, si no de "práctica profesional" docente, y se refieren al profesor como "(...) un dinamizador de posibilidades, autónomo, un intelectual atento a los requerimientos de la realidad en la que interviene, investigador de su práctica, dispuesto a transformar su acción sobre la base de la toma de decisiones producto de la reflexión sobre lo que hace, o mejor sobre lo que deja de hacer". García, Loredo y Carranza (2008), hablan de la "práctica educativa" de los docentes, y se refieren a ella como una actividad dinámica, reflexiva, que incluye todos los acontecimientos que ocurren en la interacción entre el profesor y sus alumnos. Esta práctica no se restringe solo a los procesos educativos que tienen lugar dentro del aula de clase, sino que incluye también todo tipo de intervención pedagógica que ocurre fuera del aula y las acciones que realiza el profesor antes y después de las clases.

Se percibe que, a pesar que haya esos pequeños cambios en la denominación del trabajo del profesor, realmente se refieren a lo mismo; así, la práctica pedagógica se concibe como el conjunto de acciones y de momentos que se viven dentro o fuera del aula de clase, y describen el quehacer del profesor y sus alumnos, en la búsqueda de unos objetivos de formación establecidos en el currículo, que determinan directamente el aprendizaje de sus alumnos.

Para analizar la práctica pedagógica del profesor, en este texto se usa la clasificación establecida por Porlán (1989), quien fija cuatro tendencias didácticas que caracterizarían dicha práctica, y que denomina tradicional, tecnológica, espontaneista e investigativa. La tradicional identifica al profesor como un transmisor de contenidos y al alumno como un receptor que no participa de forma activa; aquí el uso de libros de texto es el único material curricular que tiene el profesor y su meta es la evaluación de los contenidos que han sido memorizados por los alumnos; así el aprendizaje se mide como evaluación sumativa y no formativa; es decir, al aprendizaje se asigna un valor numérico que mide la habilidad que posean los alumnos para grabar información.

La tendencia tecnológica fija al profesor el seguimiento de un planeamiento previamente establecido, con objetivos puntuales; aquí el principal responsable del aprendizaje es el estudiante, quien debe seguir los planes y guías establecidos por su maestro. La tendencia espontaneísta ve al estudiante como ser autónomo y que aprende espontáneamente; se centra en los intereses de los estudiantes y la conceptualización de los contenidos no es lo más importante, sino su desarrollo personal; aquí se destacan más los procedimientos y el aprendizaje es más de carácter formativo que informativo, y busca que el estudiante sea capaz de afrontar problemas cotidianos por sí solo y por esto se busca desarrollar valores racionales; la evaluación es un proceso permanente y tiene carácter formativo.

La tendencia investigativa ve al estudiante como constructor de su propio conocimiento y el aprendizaje se basa en la indagación, el cuestionamiento permanente y la generación de la duda; es decir, la clase gira entorno a la pregunta y la búsqueda 
de la respuesta; la evaluación tiene carácter formativo, al profesor no le interesa solo el aprendizaje, fomenta actitudes positivas hacia el conocimiento y el desarrollo de los procedimientos.

\subsection{Las situaciones didácticas y las situaciones a-didácticas}

Según Brousseau (2000), debe reconocerse que: "(...) la didáctica no consiste en ofrecer un modelo para la enseñanza sino en producir un campo de cuestiones que permita poner a prueba cualquier situación de enseñanza, y corregir y mejorar las que se han producido, y formular interrogantes sobre lo que sucede".

Para Brousseau (1986), las situaciones didácticas son un medio para discutir con los maestros acerca de lo que hacen o podrían hacer, y para considerar cómo se tendrían en cuenta los resultados de las investigaciones en el área de la didáctica de la matemática. La Teoría de las Situaciones Didácticas estudia la interacción entre el profesor y sus estudiantes, en torno a un saber que se pretende que el alumno incorpore. Es una forma privilegiada para comprender lo que hacen los profesores y los alumnos, y permite examinar problemas relacionados con la apropiación de saberes y se convierte en un medio de comunicación entre los investigadores y los profesores.

En palabras de Brousseau (2000), se llama situación a un modelo de interacción de un sujeto con cierto medio, que se convierte en el recurso del que dispone para alcanzar o conservar en este medio un estado favorable para el aprendizaje. Algunas de estas situaciones requieren del dominio anterior de todos los conocimientos y esquemas necesarios, pero hay otras que ofrecen una posibilidad al sujeto para construir por sí mismo un conocimiento nuevo en un proceso genético. Así, la misma palabra situación sirve, en su sentido ordinario, para describir tanto al conjunto (no necesariamente determinado) de condiciones que enmarcan una acción, como al modelo teórico y eventualmente formal que sirve para estudiarla.
En las situaciones intervienen tres elementos fundamentales: el estudiante, el profesor y el medio didáctico. D'Amore y Fandiño-Pinilla (2002) señalan que en esta terna el profesor facilita el medio en el cual el estudiante construye su conocimiento. Cuando se refiere a las situaciones se hace alusión a tareas, actividades o prácticas educativas que se caracterizan por ser diseñadas y construidas intencionalmente por un determinado sujeto -profesor- con el fin de enseñar un concepto, noción u objeto de conocimiento a otro sujeto alumno- (Brousseau, 2000).

Es imposible concebir la enseñanza y el aprendizaje de cualquier área de conocimiento sin tener en cuenta las interacciones, interrelaciones y fenómenos que emergen entre sus tres agentes principales (Fernández-Guerrero \& González-Ferro, 2017):

El alumno, cuyo papel es aprender aquello que ha sido señalado por la comunidad educativa, según su edad, nivel y desarrollo madurativo y cognitivo.

El saber o conjunto de conocimientos institucionalizados, en nuestro caso, matemáticos, que deben ser aprehendidos por los alumnos para su futura aplicación y contextualización, tanto en la vida profesional o laboral como en situaciones cotidianas.

El profesor, en posición asimétrica al alumno, es el encargado de presentar el saber y hacer funcionar el proyecto de enseñanza de la manera más adecuada posible para que se dé el aprendizaje.

Brousseau (1986), con base en Piaget, afirma que los sujetos construyen conocimiento por adaptación a al medio en el que interactúan, el cual produce contradicciones, desequilibrios y dificultades, y el aprendizaje se manifiesta como resultado del proceso de adaptación del alumno. Postula que para todo conocimiento es posible construir una situación, sin que necesariamente apele directamente a dicho conocimiento. Para Brousseau, la producción de conocimiento en una clase se da a partir de dos tipos de interacciones: la interacción 
del alumno con la problemática y la interacción del profesor con el alumno, cuando éste la realiza con la problemática del saber matemático. Desde estos dos tipos de interacciones, Brousseau postula la necesidad de un medio, el cual es pensado por el profesor con una intencionalidad didáctica. Así, este autor define la situación a-didáctica como las interacciones entre el alumno y el medio que involucra la problemática, la cual modela una actividad de producción de conocimiento, de manera independiente de la mediación del profesor; en tanto que las interacciones del segundo tipo son las situaciones didácticas. Para Sadovsky (s.f.):

(...) el concepto de medio incluye entonces tanto una problemática matemática inicial que el sujeto enfrenta, como un conjunto de relaciones, esencialmente matemáticas, que se van modificando a medida que el sujeto produce conocimiento en el transcurso de la situación, transformando en consecuencia la realidad con la que interactúa (p.3).

\subsection{Metodología}

La investigación siguió un enfoque cualitativo, el cual busca posibilidades para conocer la realidad social desde la relación entre el sujeto estudioso y la realidad estudiada. Según Corbetta (2010), desde este enfoque, el mundo social existe solo en relación con la actuación humana. Otra característica del enfoque cualitativo, según Sampieri, Fernández y Baptista (2014), es que este tiene por finalidad "Describir, comprender e interpretar los fenómenos, a través de las percepciones y significados producidos por las experiencias de los participantes". La investigación cualitativa brinda múltiples posibilidades y formas de entender y conocer las realidades que configuran lo humano. En el mundo social existen varias realidades subjetivas construidas en la investigación, que varían en su forma y contenido entre individuos, grupos y culturas (Núñez-Pérez, 2015). Por tal razón, el investigador cualitativo parte de la premisa que el mundo social es "relativo" y solo se puede enten- der desde el punto de vista de los propios actores estudiados.

En este caso, la investigación fue del tipo investigación-acción, la cual propone las etapas secuenciadas de planeación, acción y evaluación, en forma de espiral. Según Kemmis (1988), la investigación-acción "Es una forma de búsqueda auto reflexiva, llevada a cabo por participantes en situaciones sociales, para perfeccionar la lógica y la equidad de las propias prácticas sociales o educativas".

El trabajo se llevó a cabo en una institución educativa de un municipio boyacense, con la participación de cuatro profesores de matemáticas y sus correspondientes grupos de estudiantes. La recolección de información comenzó con la observación no participante, la grabación de audio de sesiones de clases, un cuestionario de pregunta abierta aplicado a profesores y otro a estudiantes y un diario de campo de todas las sesiones de clase a las cuales se hizo acompañamiento. En este último, se resaltaron aspectos como: la metodología, la dinámica y la evaluación de la clase, la participación e interés de los alumnos e interacción entre estudiante y profesor.

Luego se conformó un grupo focal con los profesores involucrados en la investigación. Se han dado diferentes definiciones de grupo focal; sin embargo, son muchos los autores que coinciden en que éste es un grupo de discusión, guiado por un conjunto de preguntas diseñadas cuidadosamente con un objetivo particular (Beck, Bryman \& Futing, 2004). El objetivo del grupo focal es generar y analizar la interacción entre los integrantes del grupo y ver cómo se construyen grupalmente significados (Morgan, 2008). En este grupo los participantes conversaron a profundidad en torno a las prácticas pedagógicas de sí mismos, las interacciones entre docente - estudiante - saber y la implementación de situaciones a-didácticas en la clase de matemáticas. 


\section{Resultados y discusión}

Para el análisis de la información se introdujeron como categorías de análisis, los tres momentos que plantea la investigación acción, así: planeación, acción - ejecución y evaluación.

\subsection{Planeación}

La planeación siempre ha sido uno de los momentos más importantes y necesarios de una clase. Sin embargo, algunos de los docentes pasan por alto este momento, con argumentos como: "La planeación no es necesaria, los libros ya traen todo, teoría, ejemplos y talleres" (Profesor B, reunión 4 grupo focal, 04/09/2018). Otros afirman que si planean, constantemente lo hacen siempre bajo un esquema conceptual rígido, sin salirse de lo establecido por los estándares curriculares y siguiendo una estructura de planeación en el formato fijado por la institución, la cual evidencia una serie de pasos muy procedimentales que no permiten el libre desarrollo de la asignatura. Según Jiménez, Limas y Alarcón (2016), una planeación debe tener presente los intereses, fortalezas y debilidades de los estudiantes, a través de una evaluación permanente y desde aquí realizar el desarrollo de los contenidos curriculares.

Desde las observaciones a algunas de las clases de matemáticas, se evidenciaron inconsistencias tanto en el dominio disciplinar, como en la práctica, manifiesta en el desarrollo procedimental y didáctico. Por ejemplo, en una de las observaciones la docente inicia haciendo entrega de un libro por cada tres estudiantes -libro que al ser analizado por los docentes es catalogado como un texto incompleto e incoherente, si se considera la cantidad de errores conceptuales y procedimentales que contiene (Reunión 4 grupo focal, 04/09/2018)-; una vez que los chicos reciben el libro inmediatamente preguntan "Profe, ¿qué página?", no permitiendo que se rompa el contrato didáctico (D'Amore, 2006), y dejando en evidencia la metodología totalmente directiva de sus clases, la docente indica la página y sin más preguntas, ni intervenciones los niños inician el "taller". Durante el desarrollo del taller la docente pasaba por los puestos verificando que estuvieran trabajando, algunos se arriesgaban a hacer una que otra pregunta como las siguientes: “En este punto, ¿qué hay que hacer?", ¿Este punto también hay que hacerlo?", "¿este se hace igual que el anterior?" (Diario de campo, 08/05/2018). Se observa cómo estas preguntas de los alumnos son superficiales, no son muestra de comprensión; solo parecen indicar que están siguiendo un procedimiento de rutina pedido por la profesora. Aquí parece claro que la planeación de esa clase se redujo a seguir el texto, pero no al desarrollo en sí de las actividades.

Guerra-lbagué, Leguízamo-Morales y Rincón-Prada (2016), señalan que en la enseñanza de las matemáticas es común observar en los profesores diferentes tipos de prácticas, lo cual obedece, entre otras cosas, al conocimiento y a la experiencia que cada docente ha adquirido a lo largo de su vida personal y profesional. La forma como cada uno desempeña su labor es producto de lo que conoce y de lo que ha vivido, aspectos particulares que caracterizan su forma de actuar. Al respecto los docentes observados reconocen en la experiencia un elemento fundamental para desempeñar su trabajo, pues no basta el saber matemático adquirido, sino también desempeñando su labor en el aula.

\subsection{Acción-ejecución}

En las observaciones de clase se pudo evidenciar cómo el uso inapropiado del lenguaje matemático, genera en los estudiantes obstáculos didácticos para el aprendizaje; según Brousseau (2000), este es un obstáculo didáctico que el profesor genera en el aprendizaje de sus estudiantes, al intentar simplificar la situación. Este obstáculo parece originarse de su experiencia como alumno y de la enseñanza de algunos de sus profesores, y como se sabe, es un impedimento en el aprendizaje y en la construcción del nuevo conocimiento. Un ejemplo del uso inapropiado del lenguaje, al parecer para facilitar los cálculos, se percibe cuan- 
do un profesor afirma: "saca el signo de la canasta" y "pase el dos a dividir para que la $x$ quede sola" (Profesor B; grabación en audio, 13/03/2018). Con la expresión "canasta" se refería al paréntesis y con "pase el dos a dividir para que la x quede sola", se refería a dividir por dos, los dos miembros de la ecuación para despejar la incógnita x. Este tipo de lenguaje es muy habitual entre los profesores de matemáticas.

Al analizar las respuestas dadas por los estudiantes en el cuestionario, se observa coherencia con lo observado en las clases; los docentes hacen uso de procedimientos que son descritos por los estudiantes; respuestas como "el profe nos da un concepto, luego nos da un ejemplo y pide que resolvamos ejercicios" (estudiante D, cuestionario 1), muestra un procedimiento típico del profesor tradicional (Jiménez \& Gutiérrez, 2017). Sin embargo, el $90 \%$ de los estudiantes dejan ver su satisfacción ante la dinámica de las clases, lo cual se evidencia con respuestas como" (...) considero que no debería cambiar ningún aspecto, ya que el método que utiliza la profe para enseñar es adecuado o es muy bueno para un buen aprendizaje" (estudiante A, cuestionario 1, 13/03/2018).

Por su parte, los docentes en el cuestionario dejan en evidencia que algunos de ellos han creado ciertas barreras, que según Godino, Batanero y Font (2003), pueden impedir una mayor eficiencia dentro de las prácticas pedagógicas, por ejemplo, la formación que ellos recibieron y los docentes que tuvieron. Para la conformación de los nuevos saberes, la mayoría de los docentes considera que siempre parten de los conocimientos previos que tienen sus estudiantes. Sin embargo, el hecho de que no todos los estudiantes estén al mismo nivel, hace que sea un poco más complicado integrar esos saberes que ya tienen los estudiantes con los nuevos saberes; incluso una docente afirma: "hay estudiantes que pareciera que no tuvieran ningún saber previo" (profesor A, cuestionario 2, 13/03/2018); pero, los docentes son conscientes que la culpa no es del todo de los estudiantes, pues estos son víctimas del facilismo y en sí del propio sistema educativo que ha permitido que la educación en Colombia desmejore, y es difícil tratar de esquivar las consecuencias de romper la negociación entre el sistema y la comunidad; o lo que podría decirse "el contrato didáctico entre el sistema escolar y los profesores", o mejor aún el contrato escolar (D’Amore, 2008).

De otro lado, las normas y aspectos como las comisiones de evaluación y promoción, los porcentajes de pérdida en cada institución, el desinterés que se evidencia por parte de los estudiantes, las huellas que dejó el decreto 230 del año 2002 -de la promoción automática-, entre otros aspectos (García, Ortiz \& Rojas, 2016), generan en los docentes muchas tensiones. Lo anterior debido a que, aunque se hable de libertad de cátedra, estas normas son una barrera que aprovecha el estudiante y la comunidad en general, incluyendo en ocasiones al mismo docente, para escudarse y hacer lo mínimo para aprobar. Sin embargo, es necesario un cambio de actitud por parte de los docentes; es importante que sea él mismo quien a través de la reflexión sobre su práctica proponga alternativas de mejoramiento y supere las dificultades encontradas. Es decir, (re)signifique sus prácticas (Jiménez, 2002).

La metodología y la manera de evaluar de los profesores investigados deja ver rasgos característicos del modelo tradicional, pues así lo evidencian sus respuestas: "yo evalúo ejercicios muy parecidos a los vistos en clase", alejándose de lo que muchos teóricos definen como evaluación. Al respecto, Álvarez (2001), plantea "(...) en el ámbito educativo debe entenderse la evaluación como actividad crítica de aprendizaje, porque se asume que la evaluación es aprendizaje en el sentido que por ella adquirimos conocimiento".

Según Hinostroza (2004), las prácticas innovadoras se caracterizan por la utilización de las potencialidades del docente para fomentar el aprendizaje, teniendo en cuenta tres aspectos: los contenidos transversales, la ampliación de los recursos de enseñanza y la creación de condiciones de apren- 
dizaje independiente. Aunque los docentes reconozcan la importancia de renovar sus prácticas pedagógicas: "siempre trato de innovar"; también es evidente que casi nunca lo hacen: "Es muy difícil planear todos los años, ya que hay mucha papelería que llenar, además, los libros traen casi todo listo para la clase" (profesor D, cuestionario 2, 13/03/2018).

Para observar otros resultados, se presenta un escenario de aula planeado desde las situaciones a-didácticas. Se consideró una situación donde participan dos jugadores en la cual el jugador que empieza jugando debe decir un número $x$ menor que 20 y el contrincante debe dar un número 1 o 2 unidades mayor: $x+m$, (con $m<3$ ). Gana el jugador que dice 20 por primera vez.

Se agruparon los estudiantes por parejas; se hace una breve explicación sobre la situación, se les pidió que en una hoja fueran anotando cada una de las partidas y que cuando llegaran a 7 partidas miraran quién había sido el ganador. Mientras iniciaban las partidas algunos descubrieron rápidamente que quien decía 17 inmediatamente ganaba; sin embargo, más adelante descubrieron que no era suficiente. El reto final implícito del juego era encontrar una posible estrategia ganadora de juego. En esta situación se evidencia lo que dicen Chevallard, Bosh y Gascón (1997): "Cada jugador produce únicamente una serie de decisiones, no tiene ningún interés en comunicar sus estrategias. Toma el juego en un cierto estado y lo deja en otro".

Luego, los alumnos son agrupados en dos equipos que compiten el uno contra el otro. A partir de aquí la situación se convierte en didáctica. En cada grupo, se asignan letras a los alumnos. A la llamada del profesor, los dos alumnos designados por la letra nombrada van a disputar una partida en el tablero. El resto del equipo solamente observa, pero no pueden ayudarles. El equipo del Jugador ganador se pone un punto. Entre partida y partida los alumnos de un mismo equipo discuten entre ellos las mejores estrategias. El éxito de cada equi- po depende de la acción y de la comprensión que cada jugador manifiesta de las estrategias que se discuten. Así, cada uno de los grupos expuso la conclusión a la que llegaron; comentaron la estrategia que como equipo usaron y dan su punto de vista de por qué falló en algunos casos la estrategia. En esta última fase, los alumnos vuelven a una situación a-didáctica, aprenden sin intervención del profesor: a enunciar "conjeturas" (como, por ejemplo, "es necesario Jugar 17"), a discutir su validez ("yo he Jugado 17 y he perdido") y a producir explicaciones ("si él juega 17, yo sólo puedo jugar 18 o 19, en los dos casos él podrá decir 20").

Ahora se retorna a la situación didáctica. Vemos como en esta sustentación de argumentos, algunos estudiantes intentaron validar o refutar algunas conjeturas (E significa estudiante. $P$ significa profesor):

E1: siempre que se dice 17 ya se gana

E2: No porque Liyen dijo 17 y perdió

E1: Pero porque no estaba atenta, cuando Leonel dijo 18 ella debió decir 20 y dijo 19.

E2: Si, pero igual dijo 17 y perdió.

E3: Se gana con varios números; por ejemplo, con el 14, el 11 y el 8 también ganan.

P: ¿Seguros que se gana con esos números?

E3: Sí, nosotros ya verificamos.

$\mathrm{P}$ : Bueno, y ¿Qué tienen en común esos números?

E1: Que si se dice 8 , después se puede decir 11, después 14 y después 17, así se gana.

$\mathrm{P}$ : ¿será que hay un número menos a 8 que me permita llegar a 8?

E4: si, el 6. 
E6: No, porque si dice 6 el otro puede decir el 8 y ya pierde

\section{E3: sería el 5}

Varios estudiantes: sí, con el 5 da 8

\section{P: ¿Cómo saben que es el 5 ?}

E5: porque si dice 5 el otro puede decir 6 o 7, y ya se puede decir 8

P: Entonces podemos decir que ¿quien diga 5 ya gana?

Varios estudiantes: sí

P: ¿Qué operación matemática hicieron para hallar ese valor?

\section{E7: Al 8 le restamos tres}

P: ¿Es posible decir que para hallar otro número solo debemos restarle 3 ?

E5: si profe, de hecho si observamos todos van de 3 en 3

$\mathrm{P}: \mathrm{y}$ ¿Por qué creen que los números van de 3 en 3 ?, ¿por qué no de 4 en 4 ?

E8: porque como se pueden decir números mayores en 1 o 2 unidades seguiría por decir un número 3 mayor unidades.

Así fue como los estudiantes descubrieron la estrategia y además la aplicaron para evidenciar que efectivamente era la estrategia ganadora.

Finalmente, se permitió hacer conjeturas a partir de lo concluido por cada uno de los estudiantes, presentándoles las siguientes situaciones:

El mismo juego, pero el jugador que empieza jugando debe decir un número $x$ menor que 45 y el contrincante debe dar un número 1 a 6 unidades mayor: $x+m$, (con $m<7)$. Gana el jugador que dice 45 por primera vez.

ii) El mismo juego, pero el jugador que empieza jugando debe decir un número $x$ menor que 100 y el contrincante debe dar un número 1 a 11 unidades mayor: $x+m$, (con $m<12)$. Gana el jugador que dice 100 por primera vez.

Se deja un espacio nuevamente para que revisen y se inician las generalizaciones, encontrando que efectivamente se cumple la misma estrategia. Esta manipulación de las variables pueden conducir, por ejemplo, a proponer el problema de encontrar una estrategia general para una "carrera al número $\mathrm{n}$ " adicionado al número propuesto un número $\mathrm{x}<\mathrm{m}$, siendo $\mathrm{n}<\mathrm{m}$. (Chevallard, Bosh \& Gascón, 1997).

Al final, cada uno de los grupos expuso la conclusión a la que llegaron; en esta situación los grupos tenían diferentes métodos por mostrar y evidenciar que sin importar el camino habían llegado a una misma conclusión. Se permitió hacer conjeturas a partir de lo concluido por cada uno de los grupos, donde se evidencia que existen varios procedimientos que permiten llegar a una única respuesta; igualmente los estudiantes que inicialmente no llegaron a la conclusión manifestaron comprender la respuesta y reconocieron que su procedimiento carecía de coherencia.

\subsection{Evaluación}

El seguimiento a estas actividades se hizo en el grupo focal, dedicando las reuniones grupales a la reflexión del quehacer pedagógico desde el área y desde la institución. Esto buscando lo afirmado por Gibb (1997): que el propósito principal de un grupo focal es hacer que surjan actitudes, sentimientos, creencias, experiencias y reacciones en los participantes; esto no sería fácil de lograr con otros métodos; además, los grupos focales permiten obtener una multiplicidad de miradas y procesos emocionales dentro del contexto del grupo. 
En una primera reunión del Grupo Focal, el investigador citó a los participantes que decidieron conformar el grupo; en esta primera sesión se hizo una pequeña caracterización del Grupo al cual se denominó "Matesitua", nombre que entre todos se eligió; se plantearon los objetivos del Grupo y la metodología de trabajo. Se hizo un análisis a priori de cómo han sido las prácticas pedagógicas en la Institución y más específicamente en el área, reconociendo que la intervención del profesor en el aula puede ser un auténtico proceso de investigación, puesto que requiere diagnosticar los diferentes estados y movimientos de la compleja vida del aula, desde la perspectiva de quienes intervienen en ella (Pérez, 1990).

En una segunda sesión, el investigador habló sobre la Teoría de las Situaciones Didácticas, algunas definiciones y cómo su influencia podía quizás cambiar el paradigma del ejercicio. En esta sesión cada docente expresó sus experiencias con situaciones innovadoras en el aula; al respecto, un docente comentó: "cuando uno les lleva algo diferente a los estudiantes, ellos creen que es juego y no hacen nada" (Diario de campo, 08/05/2018); sin embargo, se aclara que una situación debe responder a una interacción entre el estudiante y un determinado conocimiento dado, que según Brousseau (2000), incluso algunas de estas "situaciones" requieren de la adquisición "anterior" de todos los conocimientos y esquemas necesarios, pero hay otras que ofrecen una posibilidad al sujeto para construir por sí mismo un conocimiento nuevo en un proceso "genético". Notemos que la misma palabra "situación" sirve, en su sentido ordinario, para describir tanto al conjunto (no necesariamente determinado) de condiciones que enmarcan una acción, como al modelo teórico y eventualmente formal que sirve para estudiarla (Ídem).

En una tercera sesión del Grupo Focal, se analizaron aspectos significativos encontrados en las grabaciones de las clases de cada uno de los docentes, donde se reflexionó sobre la importancia de cambiar la dinámica en las clases de matemáticas. Se hace una propuesta hacia la utilización de recursos didácticos, teniendo en cuenta que para Godino, Batanero y Font (2003), la utilización de recursos didácticos no solo hace referencia a material tangible, sino también a aquellas tareas que se proponen en la clase de matemáticas, que también hacen parte de estos recursos, puesto que al resolver estas tareas el estudiante dota de significado a los conceptos matemáticos. Por tanto, lo que se debe considerar como recurso didáctico no es solo el material concreto o visual, sino la situación didáctica integral que atiende tanto a la práctica como al discurso, de la que emergen las técnicas y estructuras conceptuales matemáticas (Jiménez, Limas \& Alarcón, 2016).

Durante la cuarta sesión, se llevó un borrador de las situaciones a desarrollar en clase y se puso a consideración de cada uno de los docentes. Estos dieron diferentes aportes a cada una de las situaciones; finalmente, se planteó qué se esperaba de cada situación, qué aporte le daba al aprendizaje de los alumnos, a qué estudiantes se llevaría y en qué momento se iban a desarrollar. En esta actividad los docentes estuvieron muy activos y motivados.

Algunos docentes agradecieron el espacio y direccionamiento de este tipo de actividades en el aula, reconociendo la importancia y validez para el aprendizaje de las matemáticas; así mismo, los docentes se comprometieron a continuar con este tipo de actividades. Al respecto, uno de los docentes comenta la siguiente experiencia:

Este tipo de actividades no solo mejoró mi práctica pedagógica en el área de matemáticas, pues como docente también de física, decidí optar por buscar actividades que motivaran a mis estudiantes. Por ejemplo, para la temática de caída libre busqué una situación que consistía en dejar caer un billete sostenido de manera vertical mientras otro intenta atraparlo rápidamente, enunciando así algunas de las teoría de la caída libre; por otro lado, para explicar electrostática, llevé a mis estudiantes una bola de papel aluminio, una bomba, un trozo de tubo pvc y un trapo, la situación con- 
sistía en frotar la bomba y el tubo con el trapo y luego acercarlo a la bola de aluminio, de ahí partí para iniciar la temática (Profesor $C$, reunión 4 grupo focal, 04/09/2018).

\subsection{Discusión}

Los resultados evidencian que el analizar actitudes y procedimientos de los docentes, a partir del análisis de sus propias prácticas pedagógicas, permite percibir la forma como se constituyen las interacciones en el aula. Para uno de los docentes, la evaluación es un ejercicio que además de determinar las fortalezas y debilidades del estudiante, permite la revisión de su propia práctica, como lo expresan García et al. (2016); para los demás es solo la confirmación que los alumnos responden exactamente $a$ un saber esperado por el docente.

En este sentido, como lo expresan Alvízar-Roldán, Estrada y Fortuny (2014), se evidencian las interacciones "del profesor con los estudiantes, entre los estudiantes, de los alumnos con los contenidos de aprendizaje y el estilo de enseñanza de cada docente". Igualmente, es necesario reconocer que: “(...) el alumno aprende adaptándose a un medio lleno de contradicciones, dificultades, desequilibrios, un poco como lo hace la sociedad humana. Este saber, fruto de la adaptación del alumno, se manifiesta por respuestas nuevas que son la prueba del aprendizaje" (Brousseau, 1986).

Esta investigación da indicios de cómo los docentes, específicamente los de matemáticas, se han dejado ver como trasmisores de contenidos a partir de la ejercitación y la repetición, características del modelo tradicional (Porlán, 1989). Lo más importante de analizar las prácticas es que se trata de un instrumento valioso para cambiar esas rutinas, para (re)significar las prácticas (Jiménez, 2002).

El automatismo en las clases de matemáticas se refleja a veces en frases intuitivas, como: "en esta ecuación, lo que está sumando pasa al otro lado a restar". Este aspecto indica las dificultades que se forjan en los alumnos, convirtiéndose en obs- táculos didácticos; cambiar exige "una toma de conciencia del proceso, la conciencia de lo que se hace y de por qué se hace" (Federici, 2004). Por ello, es necesario hacer uso de un lenguaje adecuado, desarrollar en los estudiantes un pensamiento lógico y profundizar teóricamente en los objetos matemáticos a enseñar; lo cual solo se logra si se profundiza más sobre el análisis de las prácticas en su propio contexto.

También se observó que la constante reflexión sobre el quehacer pedagógico, genera cierta intranquilidad en el docente, quien inicia la búsqueda de estrategias que le permiten mejorar su trabajo de aula. El va más allá de su preocupación por el solo desarrollo secuencial de contenidos; y cuando se inicia este tipo de práctica, se comienza a"reconocer el interés del docente por educar al estudiante no solo en conocimientos, sino también en valores... " (Jiménez, Limas \& Alarcón, 2016).

\section{Conclusiones}

La investigación permitió percibir que cuando el profesor confronta la visión de su clase con lo observado por otros y mediado por la reflexión colectiva sobre su práctica, comienza a tener otra actitud y otra forma de ver la dinámica de una clase. Cuando se implementan situaciones a-didácticas en el aula, se genera una dinámica diferente de la clase en los estudiantes, con un cambio actitudinal, mostrándose más atentos y motivados hacia el aprendizaje.

Reflexionar acerca de las prácticas pedagógicas resulta un ejercicio trascendental, no solo para los docentes, sino para todos aquellos que se encuentran inmersos en el campo educativo, ya que es un elemento que permite identificar los propósitos, las intenciones, los rasgos y las posturas que se tienen acerca de la enseñanza y del aprendizaje.

Se encontró que aquellas maneras como vivieron el aprendizaje de las matemáticas los docentes que participaron en la investigación, determinan en gran medida la manera como llevan a cabo en 
la actualidad su práctica y por tanto la forma como orientan la enseñanza. En este sentido, cabe resaltar la importancia que tiene para los docentes el gusto por la asignatura que enseñan y la manera en que ven su práctica, como una oportunidad para repetir o mejorar aquellas cosas que vivieron como estudiantes.

También se evidenció que la implementación de las situaciones a-didácticas mejora la dinámica de la clase de matemáticas; la motivación y el entusiasmo, son algunas de las emociones expresadas por los estudiantes hacia el aprendizaje, dejando en evidencia la importancia de innovar, de generar desafíos y desequilibrios, para que la enseñanza de las matemáticas sea más que solo repetir los contenidos y mecanizarlos.

El hecho de que los docentes pudieran analizar su práctica y sus actividades de enseñanza de las matemáticas, generó un espacio de reflexión que les permitió repensar e intentar transformar la dinámica de sus clases. En el caso de la evaluación, para los docentes participantes, se trata de un ejercicio que además de determinar las fortalezas y debilidades del estudiante, permite la revisión de su propia práctica; por esto, a través de la constante reflexión sobre su quehacer pedagógico, iniciaron la búsqueda de estrategias que les permitan mejorar su labor en el aula.

La participación en distintos escenarios de debate y formación docente, que permitió socializar los resultados del trabajo de investigación, generó, junto a otros docentes, espacios de reflexión y de construcción colectiva, lo cual aporta a la transformación de la enseñanza y el aprendizaje de las matemáticas. El análisis de la práctica pedagógica, para mejorarla, es un elemento valioso a considerar y queda como tema abierto de investigación en educación matemática.

\section{Referencias}

Álvarez, J. M. (2001). Evaluar para conocer, examinar para excluir. Madrid, España: Morata.
Alvízar-Roldán, M., Estrada, M., \& Fortuny, J. (2014). Actitudes del docente de matemáticas de enseñanza secundaria (ESO y Bachillerato) en la relación docente-estudiante. Barcelona, España: Universidad Autónoma de Barcelona.

Báez, M. A., Cantú, C. A., \& Gómez, K. M. (2007). Un estudio cualitativo sobre las prácticas docentes en las aulas de matemáticas en el nivel medio (Trabajo de pregrado). Universidad Autónoma de Yucatán, Mérida, México.

Beck, M., Bryman, A., \& Futing, L. (2004). The Sage Encyclopedia of Social Science Research Methods. Nueva Delhi, India: SAGE Publications

Brousseau, G. (1986). Fundamentos y Métodos de la Didáctica de la Matemática. Recherches en Didactique des Mathématiques, 7 (2), 33-115. Recuperado de: http://www.famaf.unc.edu.ar/wp-content/uploads/2015/03/BEns05.pdf

Brousseau, G. (2000). Educación y didáctica de las matemáticas. Educación Matemática, 12 (1), 5-38. Recuperado de: http://www.revista-educacion-matematica.org.mx/descargas/Vol12/1/ 03Brousseau.pdf

Cantoral, R., \& Farfán, R. (2003). Matemática Educativa: Una visión de su evolución. Revista Latinoamericana de Investigación en Matemática Educativa, 6 (1), 27-40. Recuperado de: http://www. redalyc.org/pdf/335/33560102.pdf

Chevallard, Y., Bosch, M., \& Gascón, J. (1997). Estudiar matemáticas; el eslabón perdido entre la enseñanza y el aprendizaje. Barcelona, España: ICE Universidad Autónoma/Horsori.

Corbetta, P. (2010). Metodología y técnicas de investigación cualitativa. Recuperado de: https:// diversidadlocal.files.wordpress.com/2012/09/ metodologc3ada-y-tc3a9cnicas-de-investigacic3b3n-social-piergiorgio-corbetta.pdf 
Corts, A. V., \& de la Vega, M. L. C. (2004). Matemáticas para aprender a pensar: el papel de las creencias en la resolución de problemas, Vol. 100. Madrid, España: Narcea Ediciones.

D'Amore, B. (2006). Didáctica de la Matemática. Bogotá, Colombia: Magisterio.

D’Amore, B. (2008). Epistemología, didáctica de la matemática y prácticas de enseñanza. Revista de la ASOVEMAT (Asociación Venezolana de Educación Matemática), 17 (1), 87-106. Recuperado de: http://welles.dm.unibo.it/rsddm/it/articoli/damore/655\%20Epistemologia\%20didactica\%20y\%20 practicas.pdf

D’Amore, B., \& Fandiño-Pinilla, M. I. (2002). Un acercamiento analítico al triángulo de la didáctica. Educación Matemática, 14 (1), 48-62. Recuperado de: http://www.dm.unibo.it/rsddm/it/articoli/damore/443\%20triangulo\%20de\%20la\%20didactica.pdf

Díaz, V. (2006). Formación docente, práctica pedagógica y saber pedagógico. Laurus: Revista de Educación, 12, 88-103. Recuperado de: http://www. redalyc.org/articulo.oa?id=76109906

Escobar, N. (2007). La práctica profesional docente desde la perspectiva de los estudiantes practicantes y tutores. Acción pedagógica, 16 (1), 182-193. Recuperado de: https://dialnet.unirioja.es/servlet/ articulo?codigo $=2968746$

Federici, C. (2004). Una construcción didáctica del Sistema de Numeración Decimal. Bogotá, Colombia.

Fernández-Guerrero, M. M., \& González-Ferro, V. (2017). Enseñar, el arte de transformar y crecer. Saber, Ciencia y Libertad, 12 (2), 167-174. Recuperado de: http://www.sabercienciaylibertad.org/ojs/index.php/scyl/article/view/242
García, B., Loredo, J., \&Carranza, G. (2008). Análisis de la práctica educativa de los docentes: pensamiento, interacción y reflexión. Revista electrónica de investigación educativa, 10 (SPE), 1-15. Recuperado de: http://www.scielo.org.mx/scielo.php?script=sci_arttext\&pid $=$ S1607-40412008000300006\%20 Versi\%C3\%B3n\%200n-line\%20ISSN\%2016

García, O., Ortiz, O., \& Rojas, I. (2016). Caracterización de la práctica docente en relación con la política referida a la evaluación en colegios oficiales de Bogotá. Bogotá, Colombia: Universidad de la Salle.

Gibb, A. (1997). Focus group. Social Research Update, 5 (2), 1-8. Recuperado de: sru.soc.surrey.ac.uk/ SRU19.html - 23k

Godino, J. (2003). Teoría de las funciones semióticas. Granada, España: Universidad de Granada.

Godino, J.; Batanero, C., \& Font, V. (2003). Fundamentos de la enseñanza y el aprendizaje de las matemáticas para maestros. Granada, España: ReproDigital.

Guerra-lbagué, L. M., Leguízamo-Morales, C. P., \& Rincón-Prada, D. (2016). La práctica docente en la enseñanza de las matemáticas: investigación narrativa a nueve docentes de tres instituciones educativas de Bogotá (Tesis de maestría). Universidad de la Salle, Bogotá, Colombia.

Hinostroza, J. E. (2004). Diseño de estrategias de innovación y TIC para el desarrollo de la educación. Innovar en la enseñanza y enseñar a innovar. Foco, 23. Recuperado de: http://www.expansiva. cl/media/en_foco/documentos/05052004211607. pdf

Jiménez, A., \& Gutiérrez, A. (2017). Realidades escolares en las clases de matemáticas. Educación Matemática, 29 (3), 109-129. doi: 10.24844/ EM2903.04 
Jiménez, A., Limas, L., \& Alarcón, J. (2016). Prácticas pedagógicas matemáticas de profesores de una institución educativa de enseñanza básica y media. Praxis \& Saber, 7 (13), 127 - 152. doi: https://doi. org/10.19053/22160159.4169

Jiménez-Espinosa, A. (2002). Quando professores de matemática da escola e da universidade se encontram: ressignificação e reciprocidade de saberes (Tesis doctoral). Faculdade de Educação - Universidade Estadual de Campinas (UNICAMP): Campinas SP - Brasil.

Kemmis, S. (1988). Cómo planificar la investigación acción. Barcelona: Laertes.

Ministerio de Educación Nacional, MEN. (2017). Reporte de la excelencia. Colombia. Recuperado de: https://diae.mineducacion.gov.co/dia_e/documentos/2017/115087000208.pdf

Morgan, G. (2008). Sociological Paradigms and Organizational Analysis. Londres, Inglaterra: Heinemann Educational Books.

Núñez-Pérez, V. (2015). Pedagogía social e interculturalismo: una lectura posible. Revista de Investigación, Desarrollo e Innovación, 5 (2), 141-149. doi: $10.19053 / 20278306.3716$

Pérez, A. (1990). Comprender y enseñar a comprender, reflexiones según el pensamiento de John Elliott. Elliott, J. La investigación-acción en educación. Málaga, España: Ediciones Morata

Porlán, R. (1989). Teoría del conocimiento, teoría de la enseñanza y del desarrollo profesional. Las concepciones epistemológicas de los profesores (Tesis doctoral). Universidad de Sevilla. Sevilla, España: Departamento de Didáctica de las Ciencias.

Riscanevo-Espitia, L. (2016). La teoría de la práctica social del aprendizaje en la formación de profesores de matemáticas. Revista de Investigación, Desarrollo e Innovación, 7 (1), 93-110. doi: https://doi. org/10.19053/20278306.v7.n1.2016.5635

Sadovsky, P. (s.f). La teoría de situaciones didácticas: un marco para pensar y actuar la enseñanza de la matemática. Recuperado de: https://www.fing. edu.uy/grupos/nifcc/material/2015/teoria_situaciones.pdf

Sampieri, R., Fernández, C., \& Baptista, P. (2014). Metodología de la investigación. México DF, México: Mc Graw Hill.

Serres, Y. (2007). El rol de las prácticas en la formación de docentes de matemáticas (Tesis doctoral). Instituto Politécnico Nacional, México D.F, México. Recuperado de: https://www.repositoriodigital.ipn.mx/bitstream/123456789/11430/1/serres_2007.pdf

Sola, M. (2004). La formación del profesorado en el contexto del espacio europeo de educación superior. Avances alternativos. Revista Interuniversitaria de formación del Profesorado, 18 (3), 90-105. Recuperado de: http://repository.lasalle.edu.co/ bitstream/handle/10185/1455/T85.09\%20P112a. pdf?sequence $=1$

Vesga-Bravo, G. J., \& Escobar-Sánchez, R. E. (2018). Trabajo en solución de problemas matemáticos y su efecto sobre las creencias de estudiantes de básica secundaria. Revista de Investigación, Desarrollo e Innovación, 9 (1), 103-114. doi: https://doi. org/10.19053/20278306.v9.n1.2018.8270 